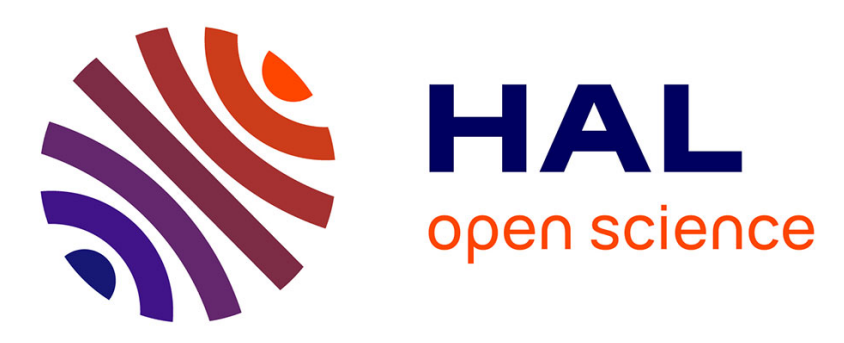

\title{
Alpha-trimmed lexicographical extrema for pseudo-morphological image analysis
}

Erchan Aptoula, Sébastien Lefèvre

\section{To cite this version:}

Erchan Aptoula, Sébastien Lefèvre. Alpha-trimmed lexicographical extrema for pseudo-morphological image analysis. Journal of Visual Communication and Image Representation, 2008, 19 (3), pp.165-174. 10.1016/j.jvcir.2007.10.001 . hal-00512672

\section{HAL Id: hal-00512672 \\ https://hal.science/hal-00512672}

Submitted on 31 Aug 2010

HAL is a multi-disciplinary open access archive for the deposit and dissemination of scientific research documents, whether they are published or not. The documents may come from teaching and research institutions in France or abroad, or from public or private research centers.
L'archive ouverte pluridisciplinaire HAL, est destinée au dépôt et à la diffusion de documents scientifiques de niveau recherche, publiés ou non, émanant des établissements d'enseignement et de recherche français ou étrangers, des laboratoires publics ou privés. 


\title{
$\alpha$-trimmed lexicographical extrema for pseudo-morphological image analysis
}

\author{
E. Aptoula*, S. Lefèvre \\ LSIIT UMR-7005 CNRS-ULP, Pôle API, Blvd Sébastien Brant, PO Box 10413, \\ 67412 Illkirch Cedex, France
}

\begin{abstract}
The extension of mathematical morphology to colour, and more generally to multivariate image data, continues to be an open problem. As its underlying theory is defined in terms of complete lattices, the main challenge lies in introducing a complete lattice structure on the image intensity range, hence vectorial extrema computation methods are necessary. In this paper, we circumvent the need for a multivariate ordering, and propose a method for directly computing the multivariate extrema of vector sets. To this end the $\alpha$-trimming principle is employed in combination with lexicographical ordering. The resulting pseudo-morphological operators, although deprived of important properties, present the advantage of a "collective" calculation, taking into account the distribution of vectors within the structuring element. They are tested against state of the art methodologies in applications treating noise reduction and texture classification, where they are shown to exhibit superior performances.
\end{abstract}

Key words: Multivariate mathematical morphology, vector ordering, vectorial processing, colour images, colour morphology

\section{Introduction}

Mathematical morphology (MM) is an algebraic image analysis framework, nowadays fully developed for binary and greyscale images. Its extension however to colour, and more generally to multivariate image data, has not yet been resolved. With the formalisation of its underlying theory during the early

* Corresponding author. Tel: +33(0)39024 44 99; fax: +33(0)39024 4455

Email address: aptoula@lsiit.u-strasbg.fr (E. Aptoula). 
1990s $[5,17,18]$, the complete lattice theory was accepted as its appropriate algebraic basis, making it possible to define morphological operators on any type of image data, as long as a complete lattice structure could be introduced on the image intensity range. In other words, at least a partial ordering is required in order to calculate the extrema of pixels. The ambiguity however of vector ordering, has been the main challenge before extending these operators to multivariate images. Several methods have been developed to this end, leading to various multivariate morphological frameworks, of which none has yet been widely accepted. Besides the inherent difficulty of ordering vectorial data, the nature of colour has also proven to be an additional obstacle in this regard. A comprehensive survey on the different approaches, not limited with lexicographical orderings, can be found in [2].

Since the ordering of vectors is a non trivial issue, the efficiency of which also depends on the application at hand, various authors have considered circumventing the need for an ordering, in the sense of a binary relation, and have concentrated directly on computing the vectorial extrema necessary for defining the fundamental dilation and erosion functions, thus leading to pseudomorphological operators. In this paper, we follow this approach and propose a modification to the widely used lexicographical ordering, based on the principle of $\alpha$-trimming, borrowed from the area of noise reduction where it has been long employed against impulsive noise in the form of the $\alpha$-trimmed mean filter and its variants [15]. The proposed $\alpha$-trimmed lexicographical extrema computation scheme, results in a flexible solution, capable of modifying the priority of vector dimensions according to its argument $\alpha$. We further discuss the choice of this parameter and propose an unsupervised computation method to this end.

Moreover, a means of transforming this type of pseudo-morphological operators into theoretically sound ones is also introduced. The practical interest of the proposed pseudo-morphological operators is illustrated in the case of colour image processing, by means of a series of comparative tests treating noise reduction and texture classification, where they exhibit a superior performance against state of the art morphological approaches.

The rest of the paper is organised as follows. Section 2 introduces briefly the theoretical aspects of extending morphological operators to multivariate images and some of the related methodologies. In section 3, the proposed approach is presented and its theoretical properties are elaborated. Then, in section 4 , we discuss the results obtained from the comparative tests. Finally section 5 is devoted to concluding remarks. 


\section{Multivariate Mathematical Morphology}

In this section we review briefly the main issues concerning the extension of MM to multivariate images, as well as some of the existing approaches and their limitations. For an in-depth study of this topic the reader can refer to the fundamental references $[5,18]$.

\subsection{Theoretical background}

After experimenting with different methods, such as the umbra approach and the threshold set approach, in order to extend binary morphology to greyscale images, the one based on complete lattices received the widest acceptance and is now considered as the right mathematical framework for MM [17]. According to this last approach, defining a complete lattice structure on the "grey levels" or intensity range of an image is sufficient for the formulation of valid morphological operators.

More precisely, given an image $f: \mathcal{E} \rightarrow \mathcal{T}$ with $\mathcal{E}$ an arbitrary non empty set, a complete lattice structure is imposed on the intensity range $\mathcal{T}$. In other words, $\mathcal{T}$ must be a non empty set equipped with a partial ordering such that every non empty subset $\mathcal{P} \subseteq \mathcal{T}$ has a greatest lower bound $\wedge \mathcal{P}$ (infimum) and a least upper bound $\bigvee \mathcal{P}$ (supremum). Consequently, the set of images $\mathcal{F}(\mathcal{E}, \mathcal{T})$ is also a complete lattice. For more details on lattice theory refer to $[4]$.

For example, as far as grey-scale images are concerned, $\mathcal{T}=\overline{\mathbb{Z}}$ or $\overline{\mathbb{R}}$ or even a finite interval $[a, b]$, hence the scalar order is employed for ordering the pixel values. However, this becomes a much more challenging task with multivariate images, where usually $\mathcal{T}=\overline{\mathbb{Z}}^{n}$ or $\mathcal{T}=\overline{\mathbb{R}}^{n}$ with $n>1$, since there is no natural ordering relation for multivariate data.

Indeed, given such a vectorial ranking scheme $<$, the vectorial versions of the two fundamental morphological operators, erosion $\left(\boldsymbol{\varepsilon}_{b}\right)$ and dilation $\left(\boldsymbol{\delta}_{b}\right)$ can be immediately derived by means of the vectorial extrema operators $\sup _{v}$ and $\inf _{v}$ based on the given ordering:

$$
\begin{aligned}
& \boldsymbol{\varepsilon}_{b}(\mathbf{f})(\mathbf{x})=\inf _{\mathbf{s} \in b}\{\mathbf{f}(\mathbf{x}+\mathbf{s})\} \\
& \boldsymbol{\delta}_{b}(\mathbf{f})(\mathbf{x})=\sup _{\mathbf{s} \in b}\{\mathbf{f}(\mathbf{x}-\mathbf{s})\}
\end{aligned}
$$

where $\mathbf{f}$ denotes a multivalued image and $b$ a flat structuring element (SE). Therefore, the main obstacle preventing the extension of morphological op- 
erators to multivalued images, consists in defining a vector ordering relation that will induce a complete lattice structure on the pixel intensity range.

\subsection{Computing vectorial extrema}

According to the classical paper of Barnett (1976) [3], most ordering approaches can be classified into the following categories:

Marginal or M-ordering, which is a partial ordering realised in a componentwise mode, hence neither exploiting the inter-channel relations nor preserving the original vectors. This last property renders marginal ordering inadequate for colour images as it introduces false colours.

Reduced or R-orderings on the other hand, first reduce vectors into scalar values, and then order them according to the natural scalar order. However, unless the reduction transformation is injective, this approach results in preorderings that do not lead necessarily to unique extrema.

Conditional or C-orderings, order vectors by means of some of their marginal components, selected sequentially according to different conditions, with the lexicographical ordering $<_{L}$ being a widely known example of this group:

$$
\forall \mathbf{v}, \mathbf{v}^{\prime} \in \mathbb{R}^{n}, \quad \mathbf{v}<_{L} \mathbf{v}^{\prime} \Leftrightarrow \exists i \in\{1, \ldots, n\}, \quad\left(\forall j<i, v_{j}=v_{j}^{\prime}\right) \wedge\left(v_{i}<v_{i}^{\prime}\right)
$$

From a theoretical point of view, lexicographical ordering is a total ordering, thus preserving the input vectors and producing unique extrema. Due to these properties as well as to its ease of customisation (i. e. changing the dimension comparison order) most efforts in the area of multivariate morphology, and colour in particular, are based upon it $[1,11]$.

\subsubsection{Improving lexicographical ordering}

Despite its theoretical advantages, the use of lexicographical ordering as defined in equation (3) is limited in practice to cases where the first dimension of the compared vectors holds the majority of the data of interest compared to the rest of the image channels. Indeed, the reason is simply the fact that the second, and remaining dimensions, do not participate in the lexicographical comparison process, unless an equality takes place in the first. Consequently, this situation leads to an inefficient exploitation of the inter-channel relations [7]. In order to remedy this problem, a first attempt was made by Ortiz et al. 
[14], that proposed the $\alpha$-lexicographical ordering:

$$
\forall \mathbf{v}, \mathbf{v}^{\prime} \in \mathbb{R}^{n}, \mathbf{v}<\mathbf{v}^{\prime} \Leftrightarrow\left\{\begin{array}{l}
v_{1}+\alpha<v_{1}^{\prime}, \text { or } \\
v_{1}+\alpha \geq v_{1}^{\prime} \text { and }\left[v_{2}, \ldots, v_{n}\right]^{T}<_{L}\left[v_{2}^{\prime}, \ldots, v_{n}^{\prime}\right]^{T}
\end{array}\right.
$$

where $\alpha \in \mathbb{R}^{+}$. The $\alpha$ argument is thus used to the end of increasing the equality probability within the first dimension, since a given scalar value $v$ becomes "equal" to all values contained in the interval $[v-\alpha, v+\alpha]$, hence allowing comparisons to reach more frequently the second dimension. Nevertheless, expression (4) is not transitive, and that is why it does not represent an ordering from an algebraic point of view. A theoretically sounder methodology was introduced by Angulo [1], the $\alpha$-modulus lexicographical ordering:

$$
\forall \mathbf{v}, \mathbf{v}^{\prime} \in \mathbb{Z}^{n}, \quad \mathbf{v}<\mathbf{v}^{\prime} \Leftrightarrow\left[\left\lceil v_{1} / \alpha\right\rceil, v_{2}, \ldots, v_{n}\right]^{T}<_{L}\left[\left\lceil v_{1}^{\prime} / \alpha\right\rceil, v_{2}^{\prime}, \ldots, v_{n}^{\prime}\right]^{T}
$$

which once more aims to create equality groups within the first dimension. It relies on a quantisation through division by a constant $\alpha$ followed by a rounding off, which reduces the dynamic margin of the first dimension, thus allowing a greater number of comparisons to reach the second.

\subsubsection{Direct computation of extrema}

Considering the ambiguity of ordering vectorial data, an alternative approach that relaxes partially this requirement consists in directly computing the extrema of a given set of vectors, without ordering them by means of a binary relation. By being able to obtain unique extrema from any given set of vectors, one can then define dilation and erosion as illustrated in equations (1) and (2) respectively, and subsequently the rest of the morphological operators.

Some examples of this approach, include the extrema obtained by cumulative distances, where the minimum of a set of vectors is defined as the median vector and the maximum as the most distant. Given $V=\left\{v_{i}\right\}$ a set of vectors:

$$
\begin{aligned}
& \max V=\arg \max _{v_{i}}\left\{\sum_{j \neq i} d\left(v_{i}, v_{j}\right)\right\} \\
& \min V=\arg \min _{v_{i}}\left\{\sum_{j \neq i} d\left(v_{i}, v_{j}\right)\right\}
\end{aligned}
$$

where $d(\cdot, \cdot)$ denotes a distance [16]. Further examples of multivariate morphology based on direct extrema calculation can be found in [10], where a graph based approach based on minimum spanning trees is employed, as well as in [9], where fuzzy subsethood degrees determine the extrema. 

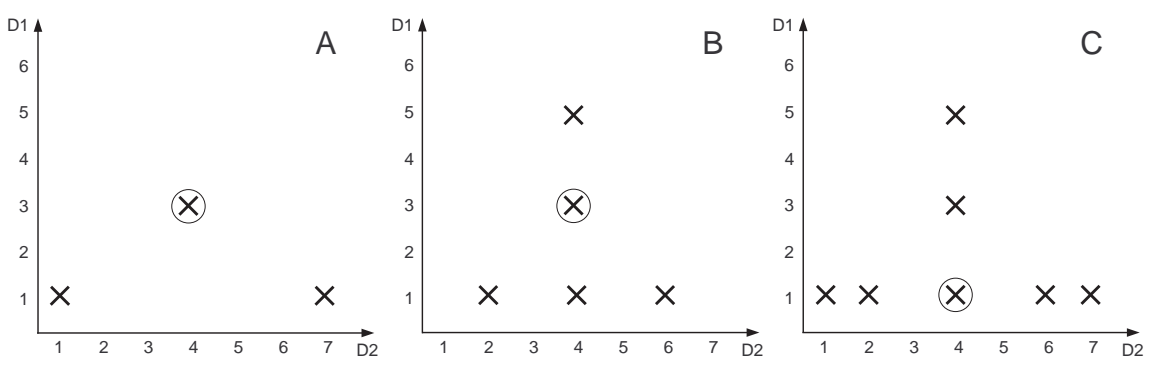

Fig. 1. From left to right, three sets of bi-dimensional vectors and their minima (circled), computed according to equation (7) using the Euclidean distance: $A=\{(1,1),(3,4),(1,7)\}$ and $\min A=(3,4), B=\{(1,2),(1,4),(3,4),(1,6),(5,4)\}$ and $\min B=(3,4)$, and $C=A \cup B$, $\min C=(1,4)$.

Nevertheless, since there is no underlying binary ordering relation, standard properties such as increasingness and idempotence can no longer be guaranteed. That is why the operators obtained in this way cannot be characterised as morphological, but rather pseudo-morphological (figure 1). Although a serious handicap, the lack of an underlying ordering relation does not hinder however the practical use of pseudo-morphological operators. On the contrary, the very "disadvantage" of a collective extremum calculation, conversely to a binary one, can be presumed to allow them to better exploit the distribution of vectors under the SE. An example of classification using multispectral remote sensing images is provided by Plaza et al. in [16], where the extended morphological profiles are calculated based on operators employing equations (6) and $(7)$.

\section{Pseudo-morphology based on $\alpha$-trimmed lexicographical extrema}

In this section, we present a means of computing the extrema of a set of vectors, based on a modified version of lexicographical ordering using the principle of $\alpha$-trimming, which results in pseudo-morphological operators.

\subsection{Definitions}

The $\alpha$-trimming principle has long been used in filters such as the $\alpha$-trimmed mean filter $(\alpha \mathrm{MF})$ and its variants [15] against impulsive noise. Given a vector $\mathbf{v} \in \mathbb{R}^{n}$, containing the sorted scalar pixels under the filtering window, the underlying idea of $\alpha$-trimming consists in computing their mean by ignoring the $2 \alpha$ extreme:

$$
\alpha \mathrm{MF}(\mathbf{v})=\frac{1}{n-2 \alpha} \sum_{i=\alpha+1}^{n-\alpha} v_{i}
$$




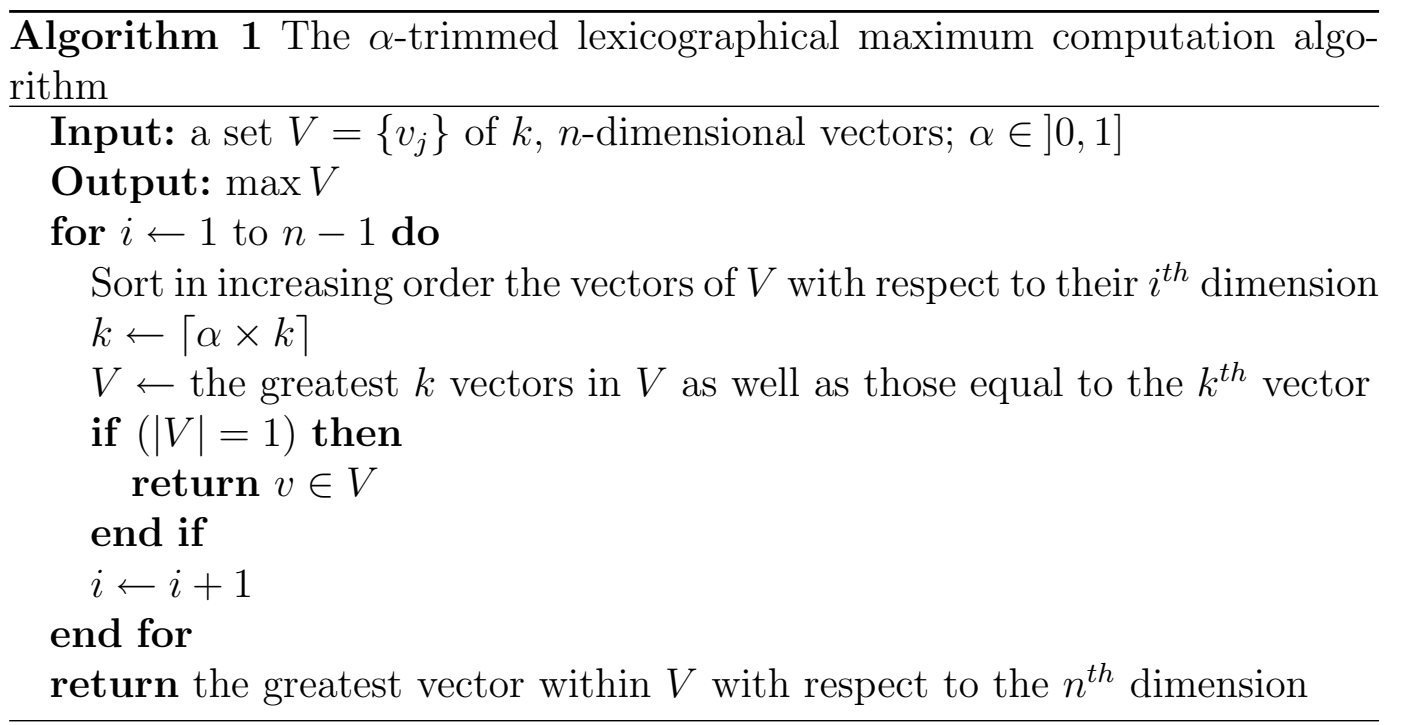

where $\alpha \in[0, n / 2]$, and $n$ is odd. Similarly, in the case of multidimensional vectors we can apply a likewise principle to each dimension in an iterative mode. Specifically, in the case of the maximum, starting from the first dimension, we can sort all $k$ vectors according to this dimension, and then keep the $\lceil\alpha \times k\rceil$ greatest. By repeating this simple process for each dimension, at each step the initial set of vectors will get smaller. In the eventual case where more than one vectors remain at the end of this procedure, the last dimension is used for determining the sought extremum. A more formal description for computing the maximum based on this procedure, is given in algorithm 1.
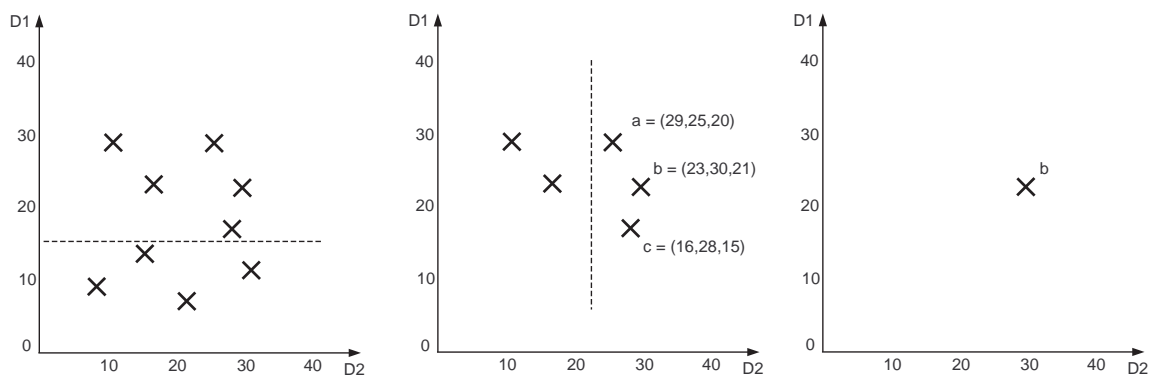

Fig. 2. A set of vectors in a three dimensional space $D 1 \times D 2 \times D 3$, and the three iterations of the $\alpha$-trimmed lexicographical maximum computation with $\alpha=0.5$ (left to right). According to the proposed approach, the maximum is the greatest, with respect to the third dimension, of the remaining three vectors $\mathbf{a}, \mathbf{b}$ and $\mathbf{c}$ in the middle.

As to the minimum, it can be obtained in a likewise mode by simply sorting in a decreasing order. Consequently, the resulting extrema can be used in order to define the operators in equations (1) and (2) for multivariate pixels. An illustration of this approach on a three dimensional space $D 1 \times D 2 \times D 3$ is given in figure 2. The "advantage" of a collective extremum calculation comes however with an increased computational burden. More precisely, assuming an optimal sorting procedure is used, in the worst scenario, where all $n$ dimensions 


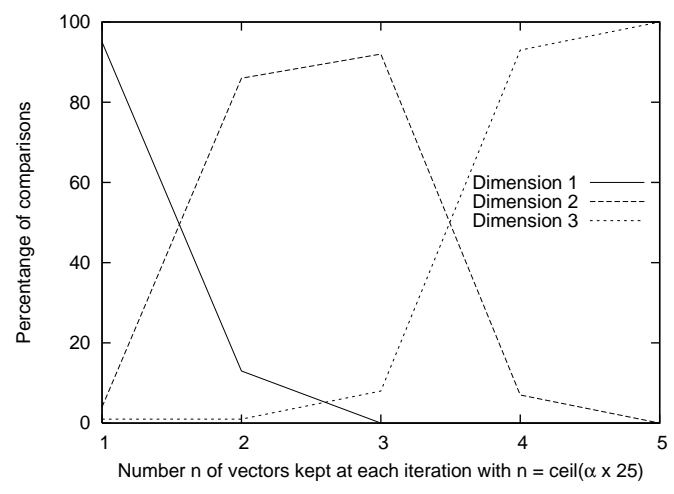

Fig. 3. The plot of comparisons decided by each dimension of the image Lenna (figure 8 left) in the I1I2I3 colour space, during a dilation with a square shaped SE of size $5 \times 5$ pixels, for various numbers of vectors kept at each iteration.

would need to be sorted, with $k$ vectors the complexity would be in the order of $O(n \times k \times \log k)$. On the other hand, with the standard lexicographical ordering and the same scenario, the complexity would be $O(n \times k)$. Note that as $k$ represents in practice the size of the SE in use, its value is relatively small.

\subsection{Setting $\alpha$}

The extrema obtained in this way, depend of course directly on the value of $\alpha$. As a matter of fact, with an $\alpha$ approaching zero, from each dimension $i$, only the extreme (i. e. maximum or minimum) vector is kept along with those equal to it with respect to dimension $i$. In other words the procedure becomes identical to the standard lexicographical ordering. On the other hand, when $\alpha$ approaches one, priority is shifted gradually to the last dimension, and almost all initial vectors reach the last dimension where the comparison is finally decided.

This transition of priority is illustrated in figure 3. When keeping only a single value at each dimension, identically to a standard lexicographical ordering, the first dimension decides the outcome of the majority of comparisons. The final decision is shifted rapidly to the last dimension by slightly increasing the number of kept vectors. The main difference with respect to other lexicographical ordering approaches, such as those presented in section 2.2.1, is the collective extremum calculation, conversely to a binary one. Specifically, even if the final extremum choice is made in the last dimension, unless $\alpha=1.0$, all previous dimensions contribute to this choice by trimming the set of vectors accordingly. Additionally, more complicated priority relations among the available channels can be established by means of different $\alpha$ values for each vector dimension.

In practice however, it is often necessary to set these arguments in an unsu- 
pervised mode. Here we introduce a simple parameter setting model based on the standard deviation $(\sigma)$ of each dimension. More precisely, if the data are relatively concentrated with respect to their $i^{\text {th }}$ dimension, or in other words if this image channel does not contain much of the total variational information, we consider using a large $\alpha$ to be more pertinent, thus decreasing the influence of this dimension by carrying the majority of the input to the next dimension with minor trimming. On the other hand, if the data are highly dispersed with respect to the other dimensions, meaning that this channel represents relatively important variational information, a small $\alpha$ would be used leading to major trimming. Given $n$ dimensions, one way of obtaining the corresponding $\alpha_{i}$ value of dimension $i$ would be:

$$
\forall i \in\{1, \ldots, n\}, \quad \alpha_{i}=1-\frac{\sigma_{i}}{\sum_{j=1}^{n} \sigma_{j}}
$$

where $\sigma_{j}$ denotes the standard deviation of dimension $j$. A more effective way of employing this principle would be to first apply a principal components transformation on the image data and then use the variances of each new dimension to this end.

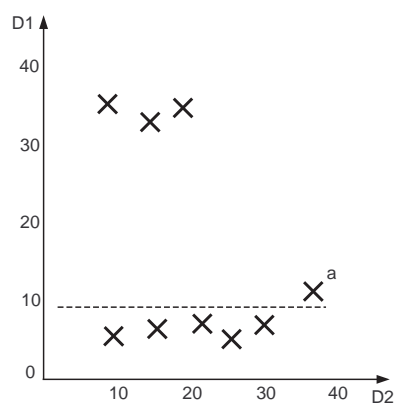

Fig. 4. Example of problematic vector distribution. The vector $\mathbf{a}$ is chosen as maximum with $\alpha=0.4$, while being "far" from the cluster of important vectors with respect to dimension D1

\subsection{Variations}

In this section, we introduce some variations to the algorithm presented in 1 . Using the $\alpha$ argument as a cardinality limit at each iteration of the algorithm can lead to undesirable situations, such as the one shown in figure 4. Indeed, by taking simply the $\lceil\alpha \times k\rceil$ extreme vectors with respect to dimension D1 the a vector is chosen as maximum, while being "far" from the cluster of important vectors with respect to the same dimension.

This situation can be easily countered by using $\alpha$ as a distance measurement. Specifically, instead of keeping the $\lceil\alpha \times k\rceil$ greatest vectors with respect to the 
$i^{\text {th }}$ dimension, one can keep only the vectors whose $i^{\text {th }}$ dimension is at most at distance $\alpha$ with respect to the greatest vector of the same dimension. Details are given in algorithm 2 .

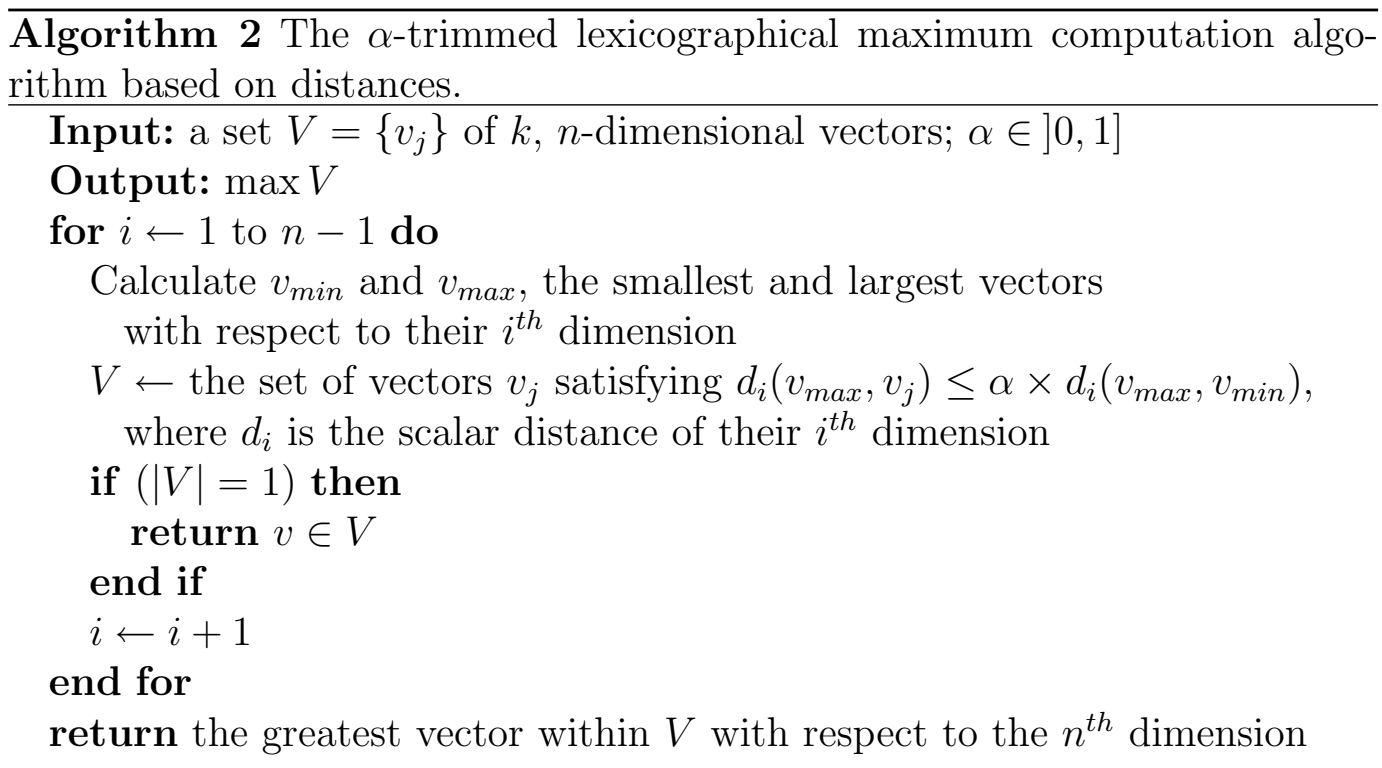

As previously mentioned, a more important "disadvantage" however of $\alpha$ trimmed lexicographical extrema is the lack of an underlying binary ordering relation. Nevertheless, given any extremum computation method, it is possible to construct from it an ordering. In particular, considering a discrete multi-dimensional space, one can employ the collective extremum computation method at hand, in order to calculate the maximum or minimum of this space, and then repeat for the remaining points, up until the entire space is ordered. The formal procedure is described in algorithm 3.

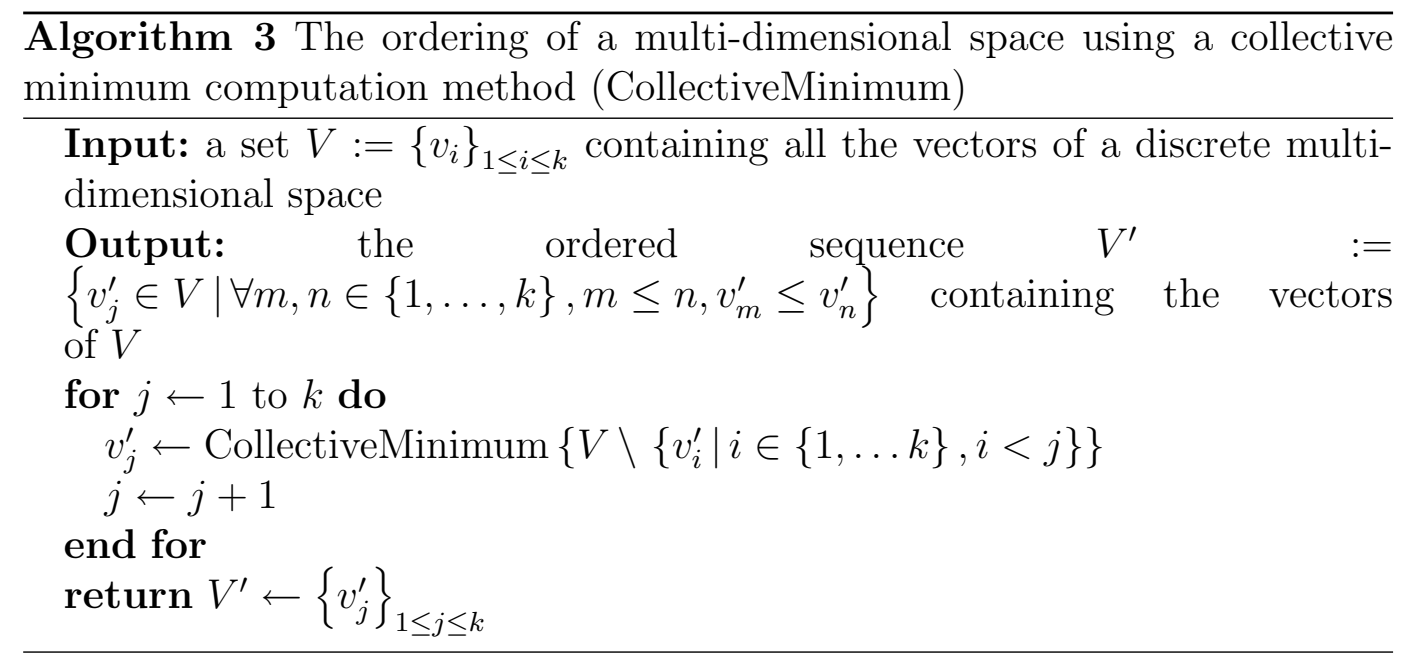

Moreover, since $\alpha$-trimmed lexicographical extrema are unique, this approach leads to a total ordering. The exact algebraic properties of the result however depend strongly both on the extremum computation method used (e.g. $\alpha$ trimmed lexicographical, cumulative distances, etc) as well as on the extremum 
employed (i. e. minimum or maximum) during its construction. Furthermore, considering its computational cost, one can also limit the procedure described in algorithm 3 only to the points of the multi-dimensional space occurring within the image to be processed, thus obtaining an "image-specific" ordering.

\subsection{Case study: colour pseudo-morphology}

The so far discussed extrema computation mechanisms have been independent of the underlying multi-dimensional space. In this section, we study the specific case of colour images, and illustrate the application of colour pseudo morphological operators in this context.

Since lexicographical ordering in general is most adequate for data spaces where an inherent priority exists among the dimensions, using the RGB colour space, where all three dimension are equally important, is considered inappropriate. Instead, an intuitive colour space based on the notions of hue $(\mathrm{H})$, saturation $(\mathrm{S})$ and luminance $(\mathrm{L})$ is chosen, the improved HLS (IHLS) space based on the max-min norm [8], which remedies important drawbacks of the standard cylindrical HLS space (e. g. dependence between saturation and luminance, etc).
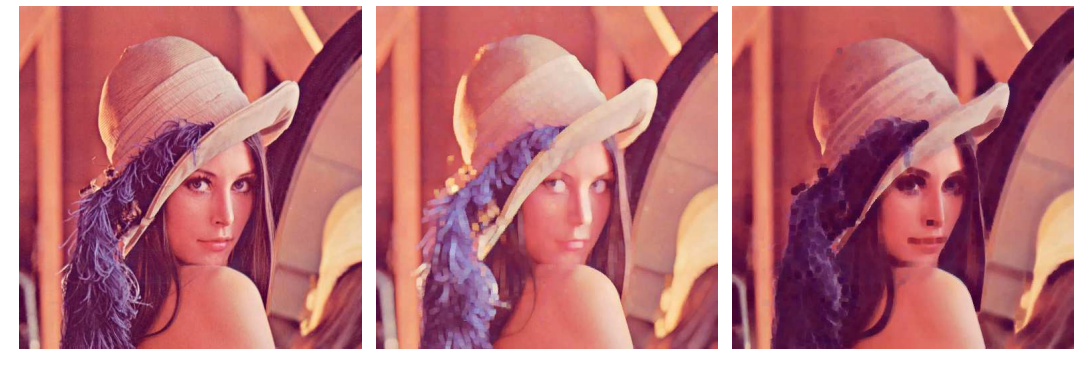

Fig. 5. From left to right, the original image Lenna, its dilation and erosion, both with a square shaped SE of size $7 \times 7$ using $\alpha$-trimmed lexicographical extrema with $\alpha$ set by equation (9) in the IHLS colour space.

Since the human vision system is largely known to attribute greater importance to brightness variations with respect to colour, it is decided to set the lexicographical comparison order as $\mathrm{L}$ followed by $\mathrm{S}$ and finally $\mathrm{H}$ where all components are normalised to $[0,1]$. As far as the periodicity of hue is concerned, its ordering is realised in terms of angular distances from a reference hue $h_{0}[6]$ :

$$
h \div h_{0}=\left\{\begin{array}{ccc}
\left|h-h_{0}\right| & \text { if } & \left|h-h_{0}\right|<0.5 \\
1-\left|h-h_{0}\right| & \text { if } & \left|h-h_{0}\right| \geq 0.5
\end{array}\right.
$$

which for the sake of simplicity is set as $h_{0}=0.0$. Image specific reference points have been additionally tested, however as the hue occupies the last position of the lexicographical cascade, their impact on the end results has 
been observed to be negligible in the context of these experiments. The hue values are then ordered according to their distances from $h_{0}$ :

$$
\forall h, h^{\prime} \in[0,1], h<h^{\prime} \Leftrightarrow h^{\prime} \div h_{0}<h \div h_{0}
$$

where hues closer to $h_{0}$ are considered greater. Consequently, the dilation and erosion operators can be defined by calculating the necessary colour extrema according to algorithm 1, using the order of dimensions as L, S and H. Figure 5 illustrates the application of the two operators on the Lenna image. The effect of $\alpha$-trimmed extrema can be better observed from figures 6 and 7 , which illustrate respectively the average values of each image channel for dilations computed based on the proposed trimmed extrema (algorithm 1) and $\alpha$-modulus lexicographical ordering (5), using varying values for $\alpha$. Note that erosions lead to symmetrical plots with respect to the horizontal axis.

a)

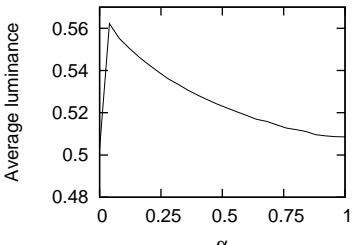

b)

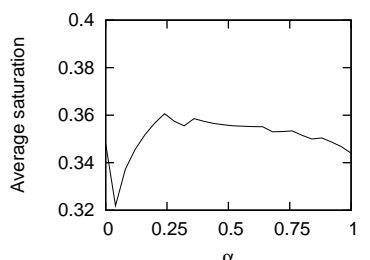

c)

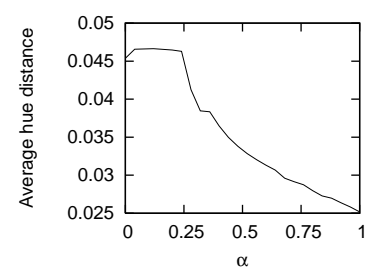

Fig. 6. The plots of average luminance (a), average saturation (b) and average hue distance (c) from $h_{0}=0.0$, computed with (10), of the Lenna image, during dilations with a square shaped SE of size $5 \times 5$ pixels using the trimmed extrema of algorithm 1 combined with varying values of $\alpha$.

a)

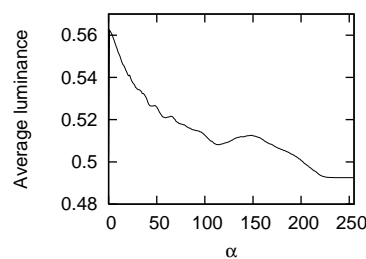

b)

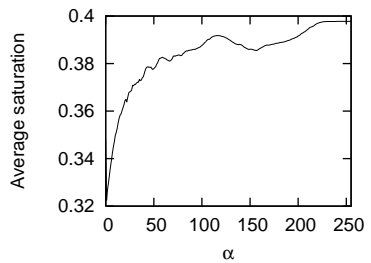

C)

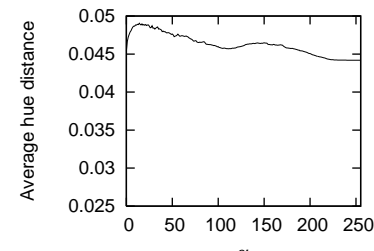

Fig. 7. The plots of average luminance (a), average saturation (b) and average hue distance (c) from $h_{0}=0.0$, computed with (10), of the Lenna image, during dilations with a square shaped SE of size $5 \times 5$ pixels using the $\alpha$-modulus lexicographical ordering (5) combined with varying values of $\alpha$.

As far as luminance is concerned (figure 6a), since small values of $\alpha$ render the ordering an approximation of its standard lexicographical counterpart, one can clearly observe a monotonic decrease in the average value, conversely to the case of $\alpha$-modulus lexicographical ordering which exhibits a more unstable behaviour (figure 7a). By increasing $\alpha$ and hence the number of vectors kept from each dimension, comparison priority is rapidly shifted towards the second channel, i. e. saturation (figure 6b). Whereas the hue (figure 6c), as the value of $\alpha$ approaches one, determines almost exclusively the outcome of vector comparisons, thus modifying the image's average distance from the reference hue accordingly. 
As to the behaviour of $\alpha$-modulus lexicographical ordering, one can remark the lower in average luminance levels (figure 7a), as well as the greater distances to the reference hue (figure $7 \mathrm{c}$ ), while saturation is much more privileged for relatively high values of $\alpha$ (figure $7 \mathrm{~b}$ ). This result is obviously due to the fact that the division taking place within the first dimension of vectors favours almost exclusively the second. Furthermore in the case of $\alpha$-modulus lexicographical ordering, also worth noting is the decrease of luminance below the initial level of the input for higher $\alpha$ values, hence leading to a darker image after dilation. As these results are provided as mere indications and are dependent on the image at hand, we further proceed to realise a series of comparative tests among the different approaches in order to better evaluate their performances.

\section{Applications}

In this section, we present the results of a series of comparative tests carried out with the purpose of both illustrating the practical advantages of the proposed extrema computation scheme with respect to state-of-the-art methodologies, as well as asserting some of the remarks made in previous sections with experimental results.

The comparison of ordering approaches in multivariate mathematical morphology is relatively problematic since their performance depends on several criteria (e. g. data space, image data, employed operators, evaluation method, etc). That is why here it has been chosen to employ two easily quantifiable tasks, colour noise reduction and texture classification. For their processing, the setup presented in section 3.4 has been used. The extrema computation approaches that have been tested are luminance only (Lum), saturation only (Sat), hue only (Hue), lexicographical (Lex), $\alpha$-lexicographical with $\alpha=0.01$ ( $\alpha$-Lex), equation (4), $\alpha$-modulus lexicographical with $\alpha=10$ ( $\alpha$ modLex), equation (5), $\alpha$-trimmed lexicographical with $\alpha=0.45$ against uncorrelated noise and $\alpha=0.15$ against correlated noise and feature extraction $(\alpha$-trimmed-Lex) or computed according to equation (9) ( $\alpha$-trimmed-adaptiveLex), and finally the total ordering resulting from the use of algorithm 3 on the IHLS space, with an $\alpha$-trimmed lexicographical minimum where $\alpha=0.45$ ( $\alpha$-trimmed-Lex-ordering) and the modified version of $\alpha$-trimmed lexicographical extrema based on distances with $\alpha=0.3$ ( $\alpha$-trimmed-Lex-distances). As a reference, the marginal ordering on RGB (MargRGB) has also been included. The arguments have been empirically set to their optimum values. 

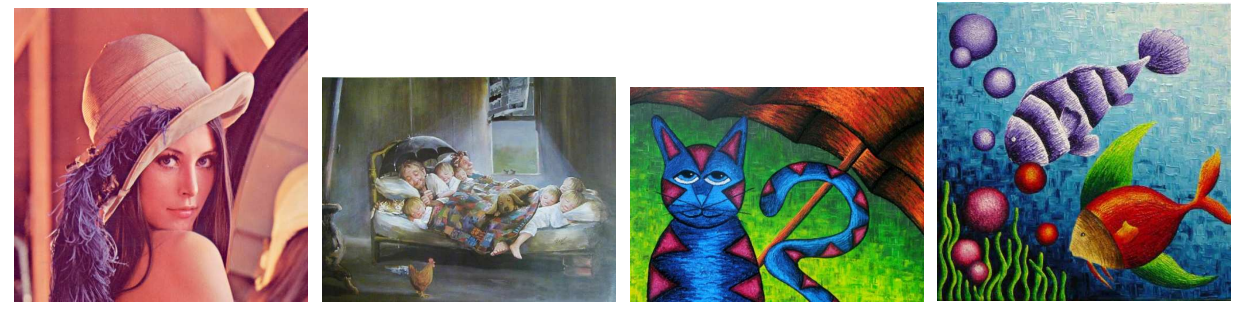

Fig. 8. From left to right, Lenna (Lenna) $512 \times 512$, the image of happiness (Happy) $552 \times 421$ (by Abidin Dino), cat with umbrella (Cat) $576 \times 420$ (by Gamze Aktan) and the curious (Curious) $502 \times 512$ (by Gamze Aktan).

\subsection{Noise reduction}

The images of figure 8 were corrupted in the RGB colour space with uncorrelated $(\sigma=0.125, \rho=0.0)$ and highly correlated zero-mean Gaussian noise ( $\sigma=0.125, \rho=0.95)$ so as to form two test sets, and in order to quantify the relative performances, the relative normalised mean squared error (RNMSE) was calculated, also in RGB:

$$
\operatorname{RNMSE}=\frac{\sum_{x, y}\left\|f(x, y)-f_{f}(x, y)\right\|^{2}}{\sum_{x, y}\left\|f(x, y)-f_{n}(x, y)\right\|^{2}}
$$

where $f(x, y), f_{n}(x, y), f_{f}(x, y)$ denote the vector pixels at position $(\mathrm{x}, \mathrm{y})$ respectively of the original, the noisy and filtered images, while $\|\cdot\|$ represents the Euclidean norm. The images were filtered using a square shaped SE of sise $(3 \times 3)$ and the open-close close-open filter $(\mathrm{OCCO})$ :

$$
\operatorname{OCCO}(f)=\frac{1}{2} \phi(\gamma(f))+\frac{1}{2} \gamma(\phi(f))
$$

where $\phi$ and $\gamma$ denote respectively the vectorial closing and opening operators.

The filtering results against uncorrelated noise are shown in table 1. Judging from the obtained values, the superiority of the marginal approach is obvious. By not being limited with the input vectors it is capable of approximating much better the original image, while the introduction of false colours is also unavoidable. Moreover, we can additionally observe the superiority of luminance over the other two dimensions, hence justifying our choice to set luminance at the first position of the lexicographical comparisons. Furthermore, one can also remark the priority attributed to the first dimension during lexicographical ordering, since its performance is very close to using only luminance. Of the first two $\alpha$ based approaches only $\alpha$-Lex leads to an improvement over Lum, with their difference however not being sufficiently large to draw sound conclusions.

Two of the four trimming based extrema on the other hand, clearly outper- 
Table 1

$100 \times R N M S E$ errors against uncorrelated Gaussian noise $(\sigma=0.125, \rho=0.0)$

\begin{tabular}{|c||c|c|c|c||c|}
\hline \multicolumn{1}{|c||}{ Method } & \multicolumn{5}{|c|}{ Images } \\
\cline { 2 - 6 } & Lenna & Happy & Cat & Curious & Average \\
\hline MargRGB & 14.07 & 14.57 & 18.44 & 24.38 & $\mathbf{1 7 . 8 7}$ \\
\hline Lum & 43.12 & 43.31 & 48.43 & 53.75 & 47.15 \\
Sat & 53.10 & 61.62 & 57.03 & 70.91 & 60.67 \\
Hue & 49.83 & 53.57 & 59.01 & 69.48 & 57.97 \\
\hline Lex & 43.20 & 43.31 & 48.50 & 53.72 & 47.18 \\
$\alpha$-Lex & 43.11 & 43.28 & 48.35 & 53.53 & 47.07 \\
$\alpha$-modLex & 43.51 & 43.92 & 48.74 & 53.79 & 47.49 \\
$\alpha$-trimmed-Lex & 29.97 & 39.74 & 35.77 & 45.16 & $\mathbf{3 7 . 6 6}$ \\
$\alpha$-trimmed-adaptive-Lex & 29.86 & 39.96 & 36.12 & 45.37 & 37.83 \\
$\alpha$-trimmed-Lex-ordering & 79.67 & 87.69 & 81.83 & 95.26 & 86.11 \\
$\alpha$-trimmed-Lex-distances & 48.48 & 44.49 & 49.27 & 55.53 & 49.51 \\
\hline
\end{tabular}

Table 2

$100 \times R N M S E$ errors against correlated Gaussian noise $(\sigma=0.125, \rho=0.95)$

\begin{tabular}{|c||c|c|c|c||c|}
\hline \multicolumn{1}{|c||}{\multirow{2}{*}{ Method }} & \multicolumn{5}{|c|}{ Images } \\
\cline { 2 - 6 } & Lenna & Happy & Cat & Curious & Average \\
\hline MargRGB & 13.94 & 14.57 & 18.65 & 24.21 & $\mathbf{1 7 . 8 4}$ \\
\hline Lum & 16.19 & 16.34 & 22.69 & 27.73 & $\mathbf{2 0 . 7 4}$ \\
Sat & 59.84 & 64.15 & 60.70 & 73.67 & 64.59 \\
Hue & 59.88 & 63.70 & 65.07 & 79.26 & 66.98 \\
\hline Lex & 16.18 & 16.33 & 22.66 & 27.70 & 20.72 \\
$\alpha$-Lex & 16.32 & 16.49 & 22.76 & 27.85 & 20.86 \\
$\alpha$-modLex & 16.98 & 17.05 & 23.22 & 28.39 & 21.41 \\
$\alpha$-trimmed-Lex & 15.94 & 16.44 & 21.94 & 27.41 & $\mathbf{2 0 . 4 3}$ \\
$\alpha$-trimmed-adaptive-Lex & 16.43 & 16.85 & 23.04 & 28.12 & 20.83 \\
$\alpha$-trimmed-Lex-ordering & 56.64 & 72.78 & 69.48 & 84.75 & 70.90 \\
$\alpha$-trimmed-Lex-distances & 17.22 & 17.00 & 23.28 & 29.16 & 21.66 \\
\hline
\end{tabular}

form their counterparts. Despite resulting in pseudo-morphological operators, collective extrema computation is presumed to aid their performance. Furthermore, although the empirically set constant $\alpha=0.45$ provides in average 
the best results, the adaptively set dimension specific arguments exhibit only slightly superior error levels. The image specific $\alpha$-trimmed lexicographical ordering as well as the distance based variation however have been disappointing, clearly showing that further work is necessary on these approaches.

As far as the case of correlated Gaussian noise is concerned (table 2), although the marginal approach continues to outperform the rest, the differences are much smaller. Besides, despite using an empirically set optimum $\alpha$ value, this time $\alpha$-trimmed lexicographical extrema hardly surpass the performance of their counterparts. A possible explanation for this result is the presence of correlated noise in its majority, within the luminance dimension, hence rendering it relatively redundant to take into account saturation and hue. Additionally, one can also observe the satisfying results obtained once more with the adaptively set arguments, asserting the robustness of the proposed model.

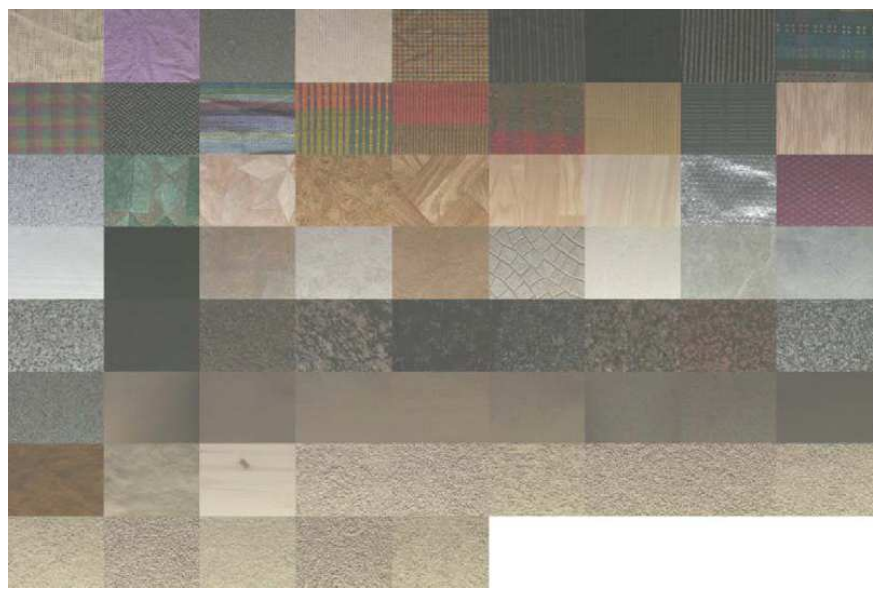

Fig. 9. Examples of the 68 textures of Outex [12].

\subsection{Texture classification}

As far as texture classification is concerned, here we employ the colour textures of Outex13 (figure 9) [13]. The question whether colour should be processed separately or jointly from texture is still an open problem [12], and vectorial morphological feature extraction operators represent in their majority the latter case. As colour texture descriptor we employ the morphological version of the autocorrelation operator, namely morphological covariance. The vectorial version of morphological covariance $K^{\prime}$ of an image $f$, is defined as the volume Vol of the image (i. e. sum of pixel values), eroded by a pair of points $P_{2, v}$ separated by a vector $\mathbf{v}$ :

$$
K^{\prime}\left(f ; P_{2, v}\right)=\operatorname{Vol}\left(\varepsilon_{P_{2, v}}(f)\right)
$$


In practice, $K^{\prime}$ is computed for varying lengths of $\mathbf{v}$, and most often, as also here the normalised version $K$ is used for measurements. Besides, since using a SE formed of only two points would be of no practical interest with trimming based approaches, here we employ $P_{2, v, b}$, a pair of $b$ SEs separated by a vector $\mathbf{v}$, with $b$ being a $3 \times 3$ cross:

$$
K(f)=\operatorname{Vol}\left(\varepsilon_{P_{2, v, b}}(f)\right) / \operatorname{Vol}(f)
$$

The covariance based feature vectors were calculated using four directions for the SE pairs $\left(0^{\circ}, 45^{\circ}, 90^{\circ}, 135^{\circ}\right)$, each along with distances ranging from 1 to 49 pixels in steps of size two. Consequently 25 values were available for each direction, making a total of 100 values for every channel after concatenation. The final image descriptor has been obtained by concatenating the marginal feature vectors of each channel, hence leading to descriptors of size 300. Table 3 contains the classification accuracy rates, computed as the fraction formed by the number of successful classifications divided by the total number of subjects.

Table 3

Classification rates in $\%$ for the textures of Outex13, using vectorial erosion based covariance.

\begin{tabular}{|c|c|}
\hline Method & Accuracy \\
\hline MargRGB & 77.65 \\
\hline Lum & 73.82 \\
Sat & 66.91 \\
Hue & 55.29 \\
\hline Lex & 74.26 \\
$\alpha$-Lex & 74.57 \\
$\alpha$-modLex & 76.62 \\
$\alpha$-trimmed-Lex & $\mathbf{8 1 . 4 7}$ \\
$\alpha$-trimmed-adaptive-Lex & 79.89 \\
$\alpha$-trimmed-Lex-ordering & 54.35 \\
$\alpha$-trimmed-Lex-distances & 73.74 \\
\hline
\end{tabular}

The overall performances appear to be relatively close. Presumably, since interchannel relations represent an important source of discriminative information as far as content description is concerned, the marginal approach loses its superiority over its vectorial counterparts. Furthermore, the pertinence of luminance over both saturation and hue is once more observed, showing that colour information is in this case just an auxiliary component. As expected, 
Lum and Lex exhibit almost identical performances, a result showing the level of priority attributed to the first component, ignoring totally the remaining two dimensions.

Minor improvements are obtained with $\alpha$-Lex and $\alpha$-modLex as they allow saturation to participate at a slightly higher degree in the process of computing covariance. However, hue remains largely unexploited. With the use of $\alpha$ trimmed extrema, the chrominance dimensions increase their contribution to the final result. As a matter of fact, the empirically set $\alpha=0.15$ also leads to an overall best, while the unsupervised model once more provides a relatively sufficient approximation. Nevertheless, no improvements are observed with the last two variations.

\section{Conclusion}

In this paper, a multivariate extrema calculation method has been presented, based on the $\alpha$-trimming principle, with the purpose of developing pseudomorphological operators. As the ambiguity of vector ordering led to a plethora of orderings approaches, the lexicographical option has been widely experimented with in this regard, as it possesses desirable theoretical properties. The proposed extremum computation scheme, aims to remedy its main drawback, which is the highly asymmetrical prioritisation of the first dimension. The use of the $\alpha$ parameter provides a flexible solution, where the contribution of each dimension to the computation outcome can be directly controlled. Moreover, a model was proposed for setting the $\alpha$ argument according to the standard deviation of each dimension.

The proposed methodology, along with its variants, has been tested against state-of-the-art ordering mechanisms used in colour morphology, in the context of noise reduction as well as texture classification. Despite empirically set arguments for all compared methodologies, $\alpha$-trimming lexicographical extrema outperformed their counterparts, with the proposed adaptive $\alpha$ calculation model also proving to be a robust and unsupervised solution for parameter setting.

Future work will concentrate on the use of $\alpha$-trimmed lexicographical extrema on multispectral imagery, as well as on more elaborate parametrisation models. In particular, the ordering derivation process will be studied, since a pertinent solution to this problem would immediately make it possible to render pseudo operators as morphologically valid. 


\section{References}

[1] J. Angulo, Unified morphological color processing framework in a lum/sat/hue representation, in: C. Ronse, L. Najman, E. Decencière (eds.), Proceedings of the 7th ISMM, vol. 30 of Computational Imaging and Vision, Springer-Verlag, Dordrecht, Germany, 2005.

[2] E. Aptoula, S. Lefèvre, A comparative study on multivariate mathematical morphology, Pattern Recognition(doi:10.1016/j.patcog.2007.02.004).

[3] V. Barnett, The ordering of multivariate data, Journal of the Statistical Society A 139 (3) (1976) 318-355.

[4] G. Birkhoff, Lattice Theory, American Mathematical Society, Providence, 1967.

[5] J. Goutsias, H. J. A. M. Heijmans, K. Sivakumar, Morphological operators for image sequences, Computer Vision and Image Understanding 62 (3) (1995) $326-346$.

[6] A. Hanbury, J. Serra, Morphological operators on the unit circle, IEEE Transactions on Image Processing 10 (12) (2001) 1842-1850.

[7] A. Hanbury, J. Serra, Mathematical morphology in the CIELAB space, Image Analysis and Stereology 21 (3) (2002) 201-206.

[8] A. Hanbury, J. Serra, Colour image analysis in 3d-polar coordinates, in: International Conference on Image Processing and its Applications, Magdeburg, Germany, 2003.

[9] M. Köppen, C. Nowack, G. Rösel, Pareto-morphology for color image processing, in: Proceedings of the 11th Scandinavian Conference on Image Analysis, vol. 1, Kangerlussuaq, Greenland, 1999.

[10] O. Lezoray, C. Meurie, A. Elmoataz, A graph approach to color mathematical morphology, in: Proceedings of the IEEE International Symposium on Signal Processing and Information Technology, Athens, Greece, 2005.

[11] G. Louverdis, M. Vardavoulia, I. Andreadis, P. Tsalides, A new approach to morphological color image processing, Pattern Recognition 35 (8) (2002) 17331741 .

[12] T. Mäenpää, M. Pietikäinen, Classification with color and texture: jointly or separately?, Pattern Recognition 37 (8) (2004) 1629-1640.

[13] T. Ojala, T. Mäenpää, M. Pietikäinen, J. Viertola, J. Kyllönen, S. Huovinen, Outex: New framework for empirical evaluation of texture analysis algorithms, in: Proceedings of the 16th ICPR, vol. 1, Quebec City, Canada, 2002.

[14] F. Ortiz, F. Torres, J. Angulo, S. Puente, Comparative study of vectorial morphological operations in different color spaces, Proceedings of Intelligent robots and computer vision XX: Algorithms, Techniques, and Active Vision 4572 (2001) 259-268. 
[15] R. Öten, R. J. P. De Figueiredo, Adaptive alpha-trimmed mean filters under deviations from assumed noise model, IEEE Transactions on Image Processing 13 (5) (2004) 627-639.

[16] A. Plaza, P. Martinez, R. Perez, J. Plaza, A new approach to mixed pixel classification of hyperspectral imagery based on extended morphological profiles, Pattern Recognition 37 (6) (2004) 1097-1116.

[17] C. Ronse, Why mathematical morphology needs complete lattices, Signal Processing 21 (2) (1990) 129-154.

[18] J. Serra, Anamorphoses and function lattices, in: E. R. Dougherty (ed.), Mathematical Morphology in Image Processing, chap. 13, Marcel Dekker, New York, 1993, pp. 483-523. 